\title{
Generalized density functional theory for degenerate states
}

\author{
Á. Nagy \\ Department of Theoretical Physics, University of Debrecen, and Atomic and Molecular Physics Research \\ Group of the Hungarian Academy of Sciences, H-4010 Debrecen, Hungary \\ S. Liu \\ Department of Academic Technology and Networks, University of North Carolina, Chapel Hill, North \\ Carolina 27599-3455 \\ L. Bartolloti \\ Department of Chemistry, East Carolina University, Greenville, North Carolina 27858
}

(Received 22 October 2004; accepted 24 January 2005; published online 7 April 2005)

\begin{abstract}
An extension of density functional theory is proposed for degenerate states. There are suitably selected basic variables beyond the subspace density. Generalized Kohn-Sham equations are derived. A direct method is proposed to ensure the fixed value of ensemble quantities. Then the Kohn-Sham equations are similar to the conventional Kohn-Sham equations. But the Kohn-Sham potential is different for different ensembles. A simple local expression is proposed for the correlation energy. (C) 2005 American Institute of Physics. [DOI: 10.1063/1.1871933]
\end{abstract}

\section{INTRODUCTION}

In a couple of recent papers ${ }^{1}$ it was shown that it is possible to choose arbitrary physical quantities as basic variables to determine the ground-state properties of the system. An approach presented by Nagy and Parr in the local thermodynamical formalism ${ }^{2}$ can be considered a special case of this general approach. Recently, Becke ${ }^{3}$ and Maximoff et al. ${ }^{4}$ treated degenerate states by a method that can also be regarded a special case of the theory of Higuchi and Higuchi. ${ }^{1}$ Here, an extension of this general theory is proposed for degenerate states.

\section{GENERALIZED HOHENBERG-KOHN THEOREM FOR A DEGENERATE STATE}

In an earlier paper one of the authors ${ }^{5}$ proposed a formalism for degenerate states. Now, we extend this theory into a more general one.

Consider the lowest-lying solutions of the Schrödinger equation

$$
\hat{H}\left|\Psi_{\gamma}^{\Gamma}\right\rangle=E_{0}^{\Gamma}\left|\Psi_{\gamma}^{\Gamma}\right\rangle \quad\left(\gamma=1,2, \ldots, g_{\Gamma}\right),
$$

where $g_{\Gamma}$ is the degeneracy. (Only one index is used to denote the symmetry both in spin and ordinary space.) The subscript 0 in the energy shows that only the lowest-lying solution of symmetry $\Gamma$ is regarded. The space of all antisymmetric wave functions is divided into disjoint subspaces with different symmetries. The constrained-search technique is applied over a subspace $S^{\Gamma}$. The dimension of the subspace is equal to the degeneracy $g_{\Gamma}$.

The subspace density is defined by

$$
n^{\Gamma}=\sum_{\gamma=1}^{g_{\Gamma}} w_{\gamma} \int\left|\Psi_{\gamma}^{\Gamma}\right|^{2} d s_{1} d \mathbf{x}_{2} \cdots d \mathbf{x}_{N},
$$

where $\mathbf{x}$ stands for both the coordinates and the spin. The weighting factors $w_{\gamma}$ should satisfy the conditions

$$
1=\sum_{\gamma=1}^{g_{\Gamma}} w_{\gamma}
$$

and

$$
w_{\gamma} \geqslant 0 \text {. }
$$

The superscript $\Gamma$ in $n^{\Gamma}$ denotes that it is constructed from wave functions that belong to the subspace $S^{\Gamma}$. The subspace densities do not generally have the symmetry $\Gamma$. Their symmetry depends on the weighting factors $w_{\gamma}$, too. In principle, any set of weighting factors $w_{\gamma}$ satisfying conditions (3) and (4) can be used. It was shown that if the weighting factors $w_{\gamma}$ are all equal, the subspace density has the symmetry of the external potential. So, for instance, for atoms the subspace density is spherically symmetric. The subspace density matrix is defined as

$$
\hat{D}^{\Gamma}=\sum_{\gamma=1}^{g_{\Gamma}} w_{\gamma}\left|\Psi_{\gamma}^{\Gamma}\right\rangle\left\langle\Psi_{\gamma}^{\Gamma}\right|
$$

Let $\mathbf{X}$ denote suitably selected quantity or quantities. ${ }^{1}$ The subspace average can be given by

$$
\mathbf{X}^{\Gamma}=\sum_{\gamma=1}^{g_{\Gamma}} w_{\gamma}\left\langle\Psi_{\gamma}^{\Gamma}|\hat{\mathbf{X}}| \Psi_{\gamma}^{\Gamma}\right\rangle
$$

where $\hat{\mathbf{X}}$ is the corresponding operator(s). In the following we omit the superscript $\Gamma$ to make the notation simpler. (We are always treating a given symmetry.)

Now the constrained search leads to 


$$
\begin{aligned}
E[n, \mathbf{X}] & =\min _{S} \sum_{\gamma=1}^{g} w_{\gamma}\left\langle\Psi_{\gamma}|\hat{H}| \Psi_{\gamma}\right\rangle \\
& =\min _{n, \mathbf{X}}\left\{\min _{S \rightarrow(n, \mathbf{X})} \sum_{\gamma=1}^{g} w_{\gamma}\left\langle\Psi_{\gamma}|\hat{H}| \Psi_{\gamma}\right\rangle\right\} \\
& =\min _{n, \mathbf{X}}\left\{F[n, \mathbf{X}]+\int n(\mathbf{r}) v(\mathbf{r}) d \mathbf{r}\right\},
\end{aligned}
$$

where the universal functional $F[n, \mathbf{X}]$ is defined as

$$
\left.F[n, \mathbf{X}]=\min _{S \rightarrow(n, \mathbf{X})} \sum_{\gamma=1}^{g} w_{\gamma}\left\langle\Psi_{\gamma}\right| \hat{T}+\hat{V}_{e e}\right)\left|\Psi_{\gamma}\right\rangle .
$$

$\hat{T}$ and $\hat{V}_{e e}$ are, respectively, the kinetic and the electronelectron repulsion operators. $v(\mathbf{r})$ denotes the local external potential.

Then the generalized Hohenberg-Kohn theorem has the form

$$
F[n, \mathbf{X}]+\int n(\mathbf{r}) v(\mathbf{r}) d \mathbf{r} \geqslant E_{0},
$$

which can be readily proved as follows:

$$
\begin{aligned}
& F[n, \mathbf{X}]+\int n(\mathbf{r}) v(\mathbf{r}) d \mathbf{r} \\
& =\min _{S \rightarrow(n, \mathbf{X})} \operatorname{tr}\left\{\hat{D}\left(\hat{T}+\hat{V}_{e e}\right)\right\}+\int n(\mathbf{r}) v(\mathbf{r}) d \mathbf{r} \\
& =\min _{S \rightarrow(n, \mathbf{X})} \operatorname{tr}\left\{\hat{D}\left(\hat{T}+\hat{V}+\hat{V}_{e e}\right)\right\} \geqslant E_{0},
\end{aligned}
$$

where the last inequality follows from the variational principle. The variational principle is valid here because lowestlying energy level of a given symmetry is taken. There is an equality if and only if the trial quantities $n$ and $\mathbf{X}$ are equal to the true quantities $n_{0}$ and $\mathbf{X}_{0}$.

\section{GENERALIZED KOHN-SHAM EQUATIONS FOR A DEGENERATE STATE}

The generalized Kohn-Sham equations can be derived by minimizing the kinetic energy keeping the ensemble quantities $n(\mathbf{r})$ and $\mathbf{X}$ fixed. The kinetic energy functional is defined as

$$
T_{s}[n, \mathbf{X}]=\min _{S \rightarrow(n, \mathbf{X})} \sum_{\gamma=1}^{g^{0}} w_{\gamma}\left\langle\Psi_{\gamma}^{0}|\hat{T}| \Psi_{\gamma}^{0}\right\rangle,
$$

where the superscript 0 refers to the noninteracting system. The following step is to express the ensemble quantities with the orbitals. As we have a degenerate state the wave function cannot be given by a single Slater determinant even in the noninteracting case. A linear combination of Slater determinants $\Phi$ has to be taken:

$$
\Psi_{\gamma}^{0}=\sum_{j}^{g^{0}} c_{j}^{\gamma} \Phi_{j}^{\gamma} .
$$

Because of the normalization of the wave function $\Psi_{\gamma}^{0}$,

$$
\sum_{j}^{g^{0}}\left|c_{j}^{\gamma}\right|^{2}=1 .
$$

Let $\phi_{i}$ denote the orbitals. The density obtained from the wave function $\Psi_{\gamma}^{0}$ is given by

$$
\begin{aligned}
n_{\gamma} & =N \int\left|\Psi_{\gamma}^{0}\right|^{2} d \mathbf{x}_{2} \cdots d \mathbf{x}_{N} d \sigma \\
& =N \int \sum_{j}^{g^{0}} \sum_{j^{\prime}=1}^{g^{0}} c_{j}^{\gamma^{*}} c_{j^{\prime}}^{\gamma} \Phi_{j}^{\gamma^{*}} \Phi_{j^{\prime}}^{\gamma} d \mathbf{x}_{2} \cdots d \mathbf{x}_{N} d \sigma \\
& =N \int \sum_{j}^{g^{0}}\left|c_{j}^{\gamma}\right|^{2}\left|\Phi_{j}^{\gamma}\right|^{2} d \mathbf{x}_{2} \cdots d \mathbf{x}_{N} d \sigma .
\end{aligned}
$$

One can immediately notice that there is no contribution from the product of different determinants. If two determinants differ only in one orbital there might be a contribution; however, it has different symmetry and corresponds to some $\gamma^{\prime}$ different from $\gamma$ :

$$
n_{\gamma}=\sum_{j}^{g^{0}}\left|c_{j}^{\gamma}\right|^{2}\left(2 \sum_{i=1}^{N_{c}}\left|\phi_{i}\right|^{2}+\sum_{i=N_{c}+1}^{M}\left|\phi_{i}\right|^{2} \Theta_{j i}^{\gamma}\right) .
$$

Orbitals in closed shells are doubly occupied. $\Theta_{j i}^{\gamma}$ is 1 if the orbital $\phi_{i}$ occurs in the determinant $\Phi_{j}^{\gamma}$, otherwise 0 . The ensemble density has the form

$$
n=\sum_{\gamma} w_{\gamma} n_{\gamma}
$$

Taking all $w_{\gamma}$ equal we obtain

$$
\begin{aligned}
n & =\sum_{\gamma} \sum_{j=1}^{g^{0}}\left|c_{j}^{\gamma}\right|^{2}\left(2 \sum_{i=1}^{N_{c}}\left|\phi_{i}\right|^{2}+\sum_{i=N_{c}+1}^{M}\left|\phi_{i}\right|^{2} \Theta_{j i}^{\gamma}\right) \\
& =2 \sum_{i=1}^{N_{c}}\left|\phi_{i}\right|^{2}+\sum_{i=N_{c}+1}^{M} \lambda_{i}\left|\phi_{i}\right|^{2},
\end{aligned}
$$

where the occupation numbers are

$$
\lambda_{i}=\sum_{j} \sum_{\gamma=1}^{g^{0}}\left|c_{j}^{\gamma}\right|^{2} \Theta_{j i}^{\gamma} .
$$

The noninteracting kinetic energy can be written as

$$
T_{s}=-\frac{1}{2} \sum_{i} \lambda_{i} \int \phi_{i}^{*} \nabla^{2} \phi_{i}
$$

where $\lambda_{i}=2$ for the closed shells. The ensemble quantity $\mathbf{X}$ should also be expressed with the orbitals. To obtain the generalized Kohn-Sham equations the noninteracting kinetic energy is minimized with the conditions that the ensemble quantities $n$ and $\mathbf{X}$ are fixed and the orbitals are orthonormals, 


$$
\begin{gathered}
T_{s}-\int \Lambda(\mathbf{r})\left(n(\mathbf{r}) d \mathbf{r}-\sum_{i} \lambda_{i}\left|\phi_{i}(\mathbf{r})\right|^{2}\right)-\int \mu(\mathbf{r})\{\mathbf{X}(\mathbf{r}) \\
\left.-\mathbf{X}\left[\phi_{i}(\mathbf{r})\right]\right\}-\sum_{i, j} \epsilon_{i j}\left(\delta_{i j}-\int \phi_{i}^{*}(\mathbf{r}) \phi_{j}(\mathbf{r})\right) d \mathbf{r},
\end{gathered}
$$

where $\Lambda(\mathbf{r}), \mu(\mathbf{r})$, and $\epsilon_{i j}$ are Lagrange multipliers. After a minimization and a unitary transformation we arrive at the one-particle equations

$$
\left[-\frac{1}{2} \nabla^{2}+\Lambda(\mathbf{r})\right] \phi_{i}+\int \mu\left(\mathbf{r}^{\prime}\right) \frac{\delta \mathbf{X}}{\delta \phi_{i}^{*}(\mathbf{r})} d \mathbf{r}^{\prime}=\varepsilon_{i} \phi_{i} .
$$

These generalized Kohn-Sham equations are much more complicated than the conventional equations.

If the ensemble quantities $\mathbf{X}$ are constants, i.e., do not depend on $\mathbf{r}$, the following direct method can be applied: the total ensemble energy and from it the Kohn-Sham potential are directly constructed with fixed value of $\mathbf{X}$. Then one does not have to use the Lagrange multipliers and the Kohn-Sham equations have the usual form. But we have different KohnSham potential and equations for every value of $\mathbf{X}$ :

$$
\left[-\frac{1}{2} \nabla^{2}+v_{K S}^{X}(\mathbf{r})\right] \phi_{i}^{X}=\varepsilon_{i}^{X} \phi_{i}^{X} .
$$

This direct method is illustrated for atomic multiplets in the following section.

\section{APPLICATION TO ATOMIC MULTIPLETS}

Using equal weighting factors for a spherically symmetric system, the ensemble noninteracting kinetic energy is given by

$$
T_{s}=\sum_{j=1}^{N} \lambda_{j} \int P_{j}\left[-\frac{1}{2} P_{j}^{\prime \prime}+\frac{l_{j}\left(l_{j}+1\right)}{2 r^{2}} P_{j}\right] d r,
$$

where $P_{j}$ and $\lambda_{j}$ are the radial ensemble orbitals and the occupation numbers corresponding to the given configuration, respectively. " denotes second derivative with respect to $r$. The radial ensemble density has the form

$$
\varrho=\sum_{j=1}^{N} \lambda_{j} P_{j}^{2} .
$$

What can we choose for the extra variables? While the current densities generally are different from zero the ensemble current density

$$
\mathbf{j}=\sum_{\gamma} w_{\gamma} \mathbf{j}_{\gamma}
$$

is zero if the weighting factors are all equal.

Instead we can select the angular momenta $L^{2}$ and $S^{2}$. Here we do not give the expectation value of the operators $L^{2}$ and $S^{2}$ with the orbitals and minimize them using Lagrange multipliers. Instead a direct method is proposed. $L^{2}$ and $S^{2}$ are constants of motion and the total ensemble energy can be given by

$$
E^{L S}=E^{L S}\left[P_{i}^{L S}\right]
$$

It can also be written as

$$
E^{L S}=E_{a v}+\sum_{j} C_{j}^{L S} B_{j}^{L S}+E_{c}^{L S},
$$

where $E_{a v}$ is the average energy of the different multiplets corresponding to a given configuration. The second term in the right-hand side of Eq. (27) is responsible for the multiplet separation and $E_{c}^{L S}$ is the correlation energy. Textbooks (see, e.g., Ref. 6) provide several methods for obtaining the expression $\Sigma_{j} C_{j}^{L S} B_{j}^{L S}$ from determinants spanning the noninteracting subspace corresponding to $L S$. The explicit form of Eq. (27) for the $p^{2}$ electron configuration: ${ }^{6}$

$$
\begin{aligned}
& E\left({ }^{3} P\right)=E_{a v}-\frac{3}{25} F^{2}(p p), \\
& E\left({ }^{1} D\right)=E_{a v}+\frac{3}{25} F^{2}(p p), \\
& E\left({ }^{1} S\right)=E_{a v}+\frac{12}{25} F^{2}(p p) .
\end{aligned}
$$

$F^{2}(p p)$ is the Slater integral,

$$
F^{2}(p p)=\iint R_{2 p}^{2}\left(r_{1}\right) R_{2 p}^{2}\left(r_{2}\right) \frac{r_{<}^{2}}{r_{>}^{3}} d r_{1} d r_{2}
$$

where $R_{2 p}$ is the radial wave function of the $2 p$ electrons. $r_{<}$ means $r_{1}$ if it is smaller than $r_{2}$ and $r_{2}$ if it is smaller than $r_{1}$, and $r_{>}$is the greater of $r_{1}$ and $r_{2}$.

As $E^{L S}$ is a functional of the ensemble radial wave functions $P_{i}^{L S}$,

$$
E^{L S}=E^{L S}\left[P_{i}^{L S}\right],
$$

the OPM (Ref. 7) or KLI (Ref. 8) methods can be applied. Here the KLI method is used. Then the Kohn-Sham equations have the form

$$
\left(-\frac{1}{2} \frac{d^{2}}{d r^{2}}+\frac{l_{i}\left(l_{i}+1\right)}{r^{2}}+v_{K S}^{L S}\right) P_{i}^{L S}=\epsilon_{i}^{L S} P_{i}^{L S} .
$$

The Kohn-Sham potential

$$
v_{K S}^{L S}=v+v_{J}^{L S}+v_{x}^{L S}+w^{L S}+v_{c}^{L S},
$$

where $v_{x}^{L S}$ and $v_{c}^{L S}$ are the exchange and the correlation potentials and the potential $w^{L S}$ is responsible for the multiplet separation. The generalization of the KLI method leads to the accurate approximations, ${ }^{9}$

$$
v_{x}^{L S}=v_{S}^{L S}+\sum_{i}\left(\epsilon_{i}^{L S}-\varepsilon_{i}^{L S}\right)\left|k_{i}^{L S}\right|^{2}
$$

and

$$
w^{L S}=\sum_{i}\left(k_{i}^{L S}\right)^{2} w_{i}^{L S}=\sum_{i}\left(k_{i}^{L S}\right)^{2} \sum_{j} C_{j}^{L S} \frac{1}{P_{i}^{L S}} \frac{d B_{j}^{L S}}{d P_{i}^{L S}},
$$

where $v_{S}^{L S}$ is the Slater potential

$$
v_{S}^{L S}(\mathbf{r})=-\frac{1}{2} \int d \mathbf{r}^{\prime} \varrho^{L S}\left(\mathbf{r}^{\prime}\right)\left|\sum_{j} k_{j}^{L S}\left(\mathbf{r}^{\prime}\right) k_{j}^{L S}(\mathbf{r})\right|^{2} /\left|\mathbf{r}-\mathbf{r}^{\prime}\right| .
$$

The functions $k_{i}^{L S}$ are defined with 
TABLE I. Hartree-Fock, generalized KLI with local Wigner correlation functional (this work) and experimental total energies for the multiplets ${ }^{3} P$, ${ }^{1} D$, and ${ }^{1} S$ of the $\mathrm{C}, \mathrm{O}, \mathrm{S}$, and $\mathrm{Si}$ atoms (in Rydberg).

\begin{tabular}{lllccc}
\hline \hline & & $\mathrm{C}$ & $\mathrm{O}$ & $\mathrm{Si}$ & $\mathrm{S}$ \\
\hline${ }^{3} P$ & HF & -75.3772 & -149.6187 & -577.7085 & -795.0095 \\
& KLI+LW & -75.6934 & -150.1166 & -578.7458 & -796.2406 \\
& Expt. & -75.6904 & -150.1346 & -578.7500 & -796.2780 \\
& & & & & \\
$D$ & HF & -75.2626 & -149.4584 & -577.6300 & -794.9042 \\
& KLI+LW & -75.5940 & -149.9805 & -578.6800 & -796.1577 \\
& Expt. & -75.5979 & -149.9900 & -578.6926 & -796.1938 \\
${ }^{1} S$ & HF & -75.0991 & -149.2219 & -577.5169 & -794.7489 \\
& KLI+LW & -75.4785 & -149.8105 & -578.5725 & -796.0199 \\
& Expt. & -75.4931 & -149.8267 & -578.6097 & -796.0759 \\
\hline \hline
\end{tabular}

$$
P_{i}^{L S}=\left(\varrho^{L S}\right)^{1 / 2} k_{i}^{L S},
$$

where $\varrho^{L S}$ is the radial electron subspace density. $\left(\epsilon_{i}^{L S}-\varepsilon_{i}^{L S}\right)$ are the differences between the Kohn-Sham and the HartreeFock one-electron energies,

$$
\epsilon_{i}^{L S}-\varepsilon_{i}^{L S}=\left\langle\phi_{i}\left|v_{x}^{L S}\right| \phi_{i}\right\rangle-\left\langle\phi_{i}\left|\hat{v}_{x}^{H F}\right| \phi_{i}\right\rangle,
$$

where $\hat{v}_{x}^{H F}$ is the Hartree-Fock exchange potential taken with the density functional orbitals.

\section{RESULTS AND DISCUSSION}

There is one unknown term in the Kohn-Sham potential (34); therefore one has to use an approximation for the correlation potential $v_{c}^{L S}$. Here, a simple local Wigner type expression is applied. ${ }^{10}$ The local Wigner correlation energy is

$$
E_{c}^{L W}[n]=\int \frac{a n}{b+r_{s}} d \mathbf{r},
$$

where $r_{s}$ is the Wigner-Seitz radius,

$$
r_{s}=(3 / 4 \pi n)^{1 / 3} \text {. }
$$

The parameters $a$ and $b$ depend on $L$ and $S$.

As an illustration total energies and multiplet separation for $\mathrm{C}, \mathrm{O}, \mathrm{Si}$, and $\mathrm{S}$ atoms are presented in Tables I and II. The results of the present method are denoted by KLI+LW. For comparison the Hartree-Fock and experimental values

TABLE II. Hartree-Fock, generalized KLI with local Wigner correlation functional (this work), and other calculated and experimental total energy differences of the terms ${ }^{1} D$ and ${ }^{1} S$ from the ${ }^{3} P$ (in Rydberg) for the C, O, S, and $\mathrm{Si}$ atoms.

\begin{tabular}{llcccc}
\hline \hline & $\mathrm{C}$ & $\mathrm{O}$ & $\mathrm{Si}$ & $\mathrm{S}$ \\
\hline${ }^{3} P-{ }^{1} D$ & HF & 0.1146 & 0.1603 & 0.0785 & 0.1053 \\
& KLI+LW & 0.0994 & 0.1361 & 0.0658 & 0.0829 \\
& Expt. & 0.0929 & 0.1446 & 0.0574 & 0.0842 \\
& Other calculations & 0.1000 & 0.1520 & & \\
& & & & & \\
${ }^{3} P-{ }^{1} S$ & HF & 0.2782 & 0.3968 & 0.1916 & 0.2606 \\
& KLI+LW & 0.2149 & 0.3061 & 0.1733 & 0.2207 \\
& Expt. & 0.1973 & 0.3079 & 0.1403 & 0.2021 \\
& Other calculations & 0.2100 & 0.3212 & & \\
\hline \hline
\end{tabular}

are also shown. We applied the following parameters: $a=-0.028592, b=0.2679$ for ${ }^{3} P, a=-0.032997, b=0.340$ for ${ }^{1} D$, and $a=-0.068263, b=1.0658$ for ${ }^{1} S$. Though the local Wigner expression is a very simple approximation, the $\mathrm{KLI}+\mathrm{LW}$ method results in considerable improvement.

Table II presents multiplet separation. For comparison the multiplet separation obtained by Bagus and Bennett ${ }^{11}$ for the $\mathrm{C}$ atom and Moser and Nesbet ${ }^{12}$ for the $\mathrm{O}$ atom are also presented. These data were obtained by configuration interaction and Bethe-Goldstone calculations. The present method leads to results of similar quality. We have to emphasize that the correlation potential applied here is the simplest possible local form that satisfy important coordinate-scaling requirements. ${ }^{13-15}$

The KLI method is a very powerful approach. However, it is a very delicate problem to find an appropriate correlation functional, i.e., a correlation functional that performs well together with the KLI exchange. In the existing approximating functionals exchange and correlation are treated together and if we change only the exchange part (into KLI) the balance between the exchange and correlation is ruined and we might receive worse results than in the exchange-only case. ${ }^{9}$ Gross and co-workers ${ }^{16}$ found that among the existing correlation functional the Colle-Salvetti correlation functional is the best. In a lot of cases KLI+Colle-Salvetti gives results better than any other existing functional. However, the multiplet separation obtained by KLI+Colle-Salvetti is worse than the exchange-only KLI. ${ }^{17}$ The study of correlation leading to correct multiplet separation is a challenge for future research. Our present approach is the first promising step in that direction.

In summary, the generalization of density functional theory presented here provides a systematic way of treating multiplets. The generalized KLI method with a simple local Wigner correlation functional leads to excellent total and multiplet separation energies.

\section{ACKNOWLEDGMENTS}

This work was partially supported by the Hungarian National Scientific Fund OTKA M041537 and the Supercomputing Laboratory of the Faculty of Natural Sciences, University of Debrecen.

${ }^{1}$ M. Higuchi and K. Higuchi, Phys. Rev. B 69, 035113 (2004); 69, 165118 (2004); J. Magn. Magn. Mater. 272-76, 659 (2004).

${ }^{2}$ Á. Nagy and R. G. Parr, Proc.-Indian Acad. Sci., Chem. Sci. 106, 217 (1994); J. Mol. Struct.: THEOCHEM 501, 111 (2000); Á. Nagy, in Reviews of Modern Quantum Chemistry, edited by K. D. Sen (World Scientific, Singapore 2002), Vol. 1, p. 413.

${ }^{3}$ A. D. Becke, J. Chem. Phys. 117, 6935 (2002).

${ }^{4}$ S. N. Maximoff, M. Ernzerhof, and G. E. Scuseria, J. Chem. Phys. 120, 2105 (2004)

${ }^{5}$ Á. Nagy, Phys. Rev. A 57, 1672 (1998).

${ }^{6}$ J. C. Slater, Quantum Theory of Atomic Structure (McGraw-Hill, New York, 1960), Vol. 1.

R. T. Sharp and G. K. Horton, Phys. Rev. 30, 317 (1953); K. Aashamar, T. M. Luke, and J. D. Talman, At. Data Nucl. Data Tables 22, 443 (1978).

${ }^{8}$ J. B. Krieger, Y. Li, and G. J. Iafrate, Phys. Lett. A 146, 256 (1990); Phys. Rev. A 45, 101 (1992); 46, 5453 (1992); Int. J. Quantum Chem. 41, 489 (1992).

${ }^{9}$ Á. Nagy, J. Phys. B 32, 2841 (1999).

${ }^{10}$ E. P. Wigner, Trans. Faraday Soc. 34, 678 (1938). 
${ }^{11}$ P. S. Bagus and B. I. Bennett, Int. J. Quantum Chem. 9, 143 (1975).

${ }^{12}$ C. M. Moser and R. K. Nesbet, Phys. Rev. A 4, 1336 (1971); 6, 1710 (1972).

${ }^{13}$ M. Levy and J. P. Perdew, Phys. Rev. A 32,2010 (1985).

${ }^{14}$ L. C. Wilson and M. Levy, Phys. Rev. B 41,12930 (1990).
${ }^{15}$ A. Görling and M. Levy, Phys. Rev. B 47, 13105 (1993); Phys. Rev. A 50, 196 (1994); Int. J. Quantum Chem. 29, 93 (1995).

${ }^{16}$ T. Grabo, T. Kreibich, S. Kurth, and E. K. U. Gross, in Strong Coulomb Correlations in Electronic Structure, Beyond the Local Density Approximation, edited by V. I. Anisimov (Gordon and Breach, Tokyo, 1999).

${ }^{17}$ E. Bene and Á. Nagy, Acta Phys. Chim. Debrecina 33, 7 (2000). 